\title{
On Current and Prospective Use of Binary Thin Multilayers in Radar Absorbing Structures
}

\author{
J. KAPELEWSKI* \\ Faculty of Electronics, Military University of Technology, S. Kaliskiego 2, 00-908 Warsaw, Poland

\begin{abstract}
The main objective of the paper is to overview of the actual and potential capabilities of some complex multilayers to enhance, and control the microwave absorption. Going through available literature data we emphasize the role of interphase coupling, and material resonances in enhancing phenomena of importance for internal scattering, diffusions, and intrinsic material absorption of propagating EM wave, especially in microwave and mm wave ranges. The theoretical background is consequently formulated in terms of transmissions line approach.
\end{abstract}

DOI: 10.12693/APhysPolA.124.451

PACS: 77.55.Nv, 81.07.Oj, 77.84.Lf, 84.40.-x

\section{Introduction}

Microwave absorbing composites are mostly used in military applications, such as reduction of radar visibility in different types of targets. This is also known as stealth technology. With the rapid development of personal electronics in use today it becomes also essential to shield circuits from unwanted interference in a broad range of electromagnetic frequencies. Furthermore, the ongoing downscaling trend in electronics requires the absorbing material to be thin, lightweight, easy to manufacture, and cheap.

At radar frequencies a reduction in reflection from an object can be achieved by one of two mechanisms: (1) absorption, and (2) cancellation. Coatings, also referred as resonant absorbers, reduce radar cross-section (RCS) by the cancellation of multiple reflections. Absorption is associated with a transfer of incident wave's energy to the material. The primary property of a radar absorbing material is to dissipate the energy of the incident wave into heat. These materials have constitutive parameters that are complex numbers.

The loss mechanisms are accounted for in the permittivity $\varepsilon$ and permeability $\mu$ of the material. When expressing complex permittivity, and permeability, the relative permittivity, and the relative permeability $\varepsilon_{\mathrm{r}}, \mu_{\mathrm{r}}$, which are normalized by the free space values $\varepsilon_{0}, \mu_{0}$ are used.

It is well known that material's conductivity, complex permittivity, and permeability are frequency dependent parameters that govern the propagation of electromagnetic waves inside of the material. The engineering challenge is to design an absorber that provides low reflectivity in a wide range of microwave frequencies, and is lightweight, cheap, and easy to produce. Stacking materials with different permittivities/permeabilities on top of each other is an effective way to build a broadband

*e-mail: jerzy.kapelewski@wat.edu.pl radar absorbing materials (RAM). However, for classical materials the trade-off is the dramatic increase of RAM's thickness, and weight. Nanomaterial due to their high aspect ratio, and unique electrical, and mechanical properties is considered to be a promising alternative.

We consider a geometry where the material parameters are constants as functions of $x$ and $y$, but may depend on $z$, which is considered as the main propagation direction. This corresponds to a laminated structure, $z$ being the lamination direction.

The best known are both purely dielectric absorbers with a magnetic permeability equal to unity, and magnetic dielectrics with both dielectric $\varepsilon^{\prime}$ and magnetic $\mu^{\prime}$ permeabilities differing from unity [1]. Absorbing coatings may consist of one, two, or several layers of different materials or may be graded.

Non-conducting wideband absorbing materials are important for improving immunity of electronic equipment, and solving various problems of electromagnetic compatibility. Application of absorbing materials for the design of shielding enclosures, coatings or filtering patches is preferable, compared to metal structures for several reasons. Typically enclosures of electronic equipment are made of highly conducting metal to achieve the required shielding levels. However, requirements to make electronic devices of smaller size and weight necessitate substitution of metal by electrically conducting composites and polymers. Moreover, if a conducting surface has sharp edges, slots, and apertures, currents on this surface may drive unintentional antennas and enhance noise coupling paths as culprits of undesirable emissions [2]. One of the ways to eliminate this problem is to use absorbing materials, including applications of magneto-dielectric composite materials for electromagnetic shielding purposes.

For applications in high-speed digital electronic designs, the materials that would absorb electromagnetic energy in RF, microwave, and $\mathrm{mm}$ wave bands, are of special interest. The necessity of using absorbing materials becomes more and more important, since operating frequencies of electronic devices increase, package 
density grows dramatically, and the number of electronic equipment and devices of mass production continues to increase. Thin absorbing noise-suppressing composite sheets and coats are of special interest for such applications, especially because of miniaturization trends, and of convenience to apply directly on the surfaces to be protected, as well as their broadband performance.

The efficiency of magneto-dielectric absorbers is high because the real parts $\left(\varepsilon^{\prime}\right.$ and $\left.\mu^{\prime}\right)$ of their complex dielectric $\left(\varepsilon=\varepsilon^{\prime}-\mathrm{j} \varepsilon^{\prime \prime}\right)$ and magnetic $\left(\mu=\mu^{\prime}-\mathrm{j} \mu^{\prime \prime}\right)$ permeabilities differ from unity, and are, occasionally, large, and the imaginary parts $\left(\varepsilon^{\prime \prime}\right.$ and $\left.\mu^{\prime \prime}\right)$ differ from zero. As a result, the coating thickness being on the order of the wavelength in the material $\left(\lambda / \sqrt{\varepsilon^{\prime} \mu^{\prime}}\right)$, decreases by $\sqrt{\varepsilon^{\prime} \mu^{\prime}}$ times, and the dielectric $\varepsilon^{\prime \prime}$ and magnetic $\mu^{\prime \prime}$ losses ensure even large absorption of the incident radiation.

In our opinion, the most promising microwave absorbing materials are magneto-dielectrics. The magnetic component of absorption, $\mu^{\prime \prime}$, allows one to improve matching, broaden the range of working frequencies, increase attenuation, and reduce the thickness of the protective coating $[3,4]$.

For passive absorbers, a wider bandwidth may be obtained by utilizing multiple resistive layers, as in the Jaumann absorber; in a similar manner, the tuning range of the active Salisbury screen may be extended by incorporating multiple controllable frequency selective surface (FSS) layers, and a typical result for the two FSS case, together with the two layer passive case reported in [5].

It should be noticed that last layer could be air, or a perfect electric conductor (PEC) as a function of the absorber, we are interested to optimize. Usually in typical RAM application PEC is applied since is assumed that such absorber needs to be applied like a coating on an existing metal based structure.

One particular area in which microfabrication technology can have a definitive impact is the design of wideband electromagnetic wave absorbers. A number of applications, such as stealth technologies for RCS reduction and control, critically depends on the performance of antireflection composites. Traditional designs of electromagnetic absorbers described in the open literature include the Salisbury/Dallenbach screens, and Jaumann absorbers, as well as ferrite-based absorbers. The main limitations of classical designs are narrowband performance, and their dependence on geometric properties of the coated surface. The greater flexibility in the design of electromagnetic wave absorbers can be achieved through the addition of extra degrees of freedom on the constitutive parameters, e.g., magneto-electric effects [6].

Some new advanced absorbers represent another equally important area that can benefit from using magneto-dielectric composites and metamaterials, being capable to reducing the radar cross-section of large objects [7].

The ability to optimize the design parameters of these thin planar composite metallo- and magneto-dielectric structures allows for the synthesis of both, low-loss matched impedance magneto-dielectric layers for planar antenna miniaturization, and high-loss matched impedance layers for absorber applications with precise control of the homogeneous bulk material properties, and operation frequency [8].

\section{Exemplary material resonances of use in RAM}

\subsection{Resonance-type dielectric behavior in ferroelectric materials}

The low frequency "background" dielectric loss and the microwave dielectric loss proportional to frequency are consequences of the same resonance interpretation. The monotonic increase of dielectric loss with temperature is viewed as a manifestation of anharmonic lattice vibrations (multiphonon processes), and thermal modification of the optically active lattice vibrations. It is interesting that impurities and imperfections introduce electric moments which cause large increases in the "background" losses at low temperatures and low frequencies, as well as introducing strong coupling to the acoustic spectrum. Then the resonance description implies that these dielectric losses are of a general nature, forming a useful supplement to the "classical" models of relaxation dispersions.

\subsection{Magnetic materials}

Magnetic absorbers have been based on carbonyl iron and hexaferrites. These materials have absorbing resonances in the $\mathrm{MHz}$ and $\mathrm{GHz}$ ranges. The resonance frequency is also related to particle size.

To a large extent, the theoretical and experimental efforts designed to provide microwave absorbers are associated with two important features of their electromagnetic properties. The first is based on enhanced magnetic losses due to, for example, resonance phenomenon in the higher frequency region ( $>300 \mathrm{MHz}$ ). Additionally, it has been argued that the second feature is related to enhanced dielectric losses. Considerable research has been devoted to developing artificial composite media using a wide range of polymers filled with $M$-type hexaferrites possessing large tunable anisotropy field [9, 10]. Advances in ferrite technology have given experimentalists a control over gyromagnetic resonance characteristics for selective frequencies or over a narrow frequency range, paving the way to fabrication of radar absorbers [11].

\section{3. "Gyromagnetic" resonance}

In general, when an EM wave is irradiated into a ferromagnetic, the gyromagnetic effect leads to resonance, which is called a "natural resonance". In a ferromagnetic material with a magnetic anisotropy, the direction of magnetization is restricted around the magnetic easy-axis. Once an external magnetic field tilts the magnetization, then the magnetization starts to precess around the easy-axis due to the gyromagnetic effect. When this precession of magnetization resonates with an applied 
EM wave, a natural resonance occurs, and EM wave absorption is observed [12]. The natural resonance frequency $\left(f_{\mathrm{r}}\right)$ is proportional to the magneto-crystalline anisotropy $\left(H_{\mathrm{a}}\right)$, which is expressed by $f_{\mathrm{r}}=(\gamma / 2 \pi) H_{\mathrm{a}}$, where $\gamma$ is the gyromagnetic ratio. Common magnetic materials such as spinel ferrite show EM wave absorption in a few $\mathrm{GHz}$ region. These materials can be tuned to absorb at higher frequencies $(5-20 \mathrm{GHz})$ based on particle size.

\section{Some absorbing properties of multilayered magneto-dielectric RAS}

\subsection{Matched impedance of thin composite magneto-dielectric layers}

It is of importance that the power of the wave in magneto-dielectric material decays exponentially with distance, $x$, by the factor $\exp (-\alpha x)$. $\alpha$ is the attenuation constant of the material, and can be expressed as [13]:

$$
\alpha=-\sqrt{\mu_{0} \varepsilon_{0}} \omega\left(a^{2}+b^{2}\right)^{1 / 4} \sin \left(\frac{1}{2} \tan ^{-1}\left(-\frac{a}{b}\right)\right),
$$

where $a=\varepsilon_{\mathrm{r}}^{\prime} \mu_{\mathrm{r}}^{\prime}-\varepsilon_{\mathrm{r}}^{\prime \prime} \mu_{\mathrm{r}}^{\prime \prime}$, and $b=\varepsilon_{\mathrm{r}}^{\prime} \mu_{\mathrm{r}}^{\prime \prime}+\varepsilon_{\mathrm{r}}^{\prime \prime} \mu_{\mathrm{r}}^{\prime}$.

To get a large amount of attenuation in a small thickness $\alpha$ must be rather large, which implies that $\varepsilon_{\mathrm{r}}^{\prime}, \varepsilon_{\mathrm{r}}^{\prime \prime}, \mu_{\mathrm{r}}^{\prime}$ and $\mu_{\mathrm{r}}^{\prime \prime}$ must also be large. It was noticed [14] that this condition must be tempered with the condition, describing the reflection coefficient at an interface

$$
r=\frac{\eta_{\mathrm{S}}-\eta_{0}}{\eta_{\mathrm{S}}+\eta_{0}}=\frac{Z_{\mathrm{S}}-Z_{0}}{Z_{\mathrm{S}}+Z_{0}}
$$

with $\eta$ - admittance of the propagating medium (subscript 0 for incident medium or air, and $\mathrm{S}$ for the substrate) $(Z=1 / \eta)$, where large values of permittivity, and permeability would result in a large reflection coefficient.

The surface impedance encountered by a propagating plane wave normally incident upon the composite structure can be expressed in the form

$$
Z_{\mathrm{S}}=R_{\mathrm{S}}+\mathrm{j} X_{\mathrm{S}}
$$

Moreover, a thin slab of homogeneous magneto-dielectric layer, backed by PEC, with thickness $d$, permeability $\mu_{\mathrm{r}}$ (where $\mu_{\mathrm{r}}=\mu_{\mathrm{r}}^{\prime}-\mathrm{j} \mu_{\mathrm{r}}^{\prime \prime}$ ), and permittivity $\varepsilon_{\mathrm{r}}$ (where $\left.\varepsilon_{\mathrm{r}}=\varepsilon_{\mathrm{r}}^{\prime}-\mathrm{j} \varepsilon_{\mathrm{r}}^{\prime \prime}\right)$ would have the surface impedance

$$
Z_{\text {match }}=Z_{0} Z_{\mathrm{r}} \tanh \left(\mathrm{j} \omega \sqrt{\mu_{0} \varepsilon_{0}} n_{\mathrm{s}} d\right),
$$

where $Z_{0}$ is the vacuum impedance.

Considering the scenario where the surface impedance of the PEC backed magneto-dielectric material is matched to free space $\left(\mu_{\mathrm{r}}=\varepsilon_{\mathrm{r}}\right)$ such that $Z_{\mathrm{r}}=1$, and (3.4) simplifies to

$$
Z_{\text {match }}=Z_{0} \tanh \left(\mathrm{j} \beta_{0} n_{\mathrm{s}} d\right) \text {, }
$$

with $\beta_{0}=\omega \sqrt{\mu_{0} \varepsilon_{0}}$ and $n_{\mathrm{s}}=\sqrt{\mu_{\mathrm{r}} \varepsilon_{\mathrm{r}}}$ is the index of refraction.

Setting $Z_{\mathrm{S}}$ equal to $Z_{\text {match }}$ using (3.3), and (3.5), respectively, one can obtain [15]:

$$
\tanh \left(\mathrm{j} \beta_{0} n_{\mathrm{s}} d\right)=\frac{Z_{\mathrm{S}}}{Z_{0}} .
$$

Solving (3.6) for $n_{\mathrm{s}}$ yields

$$
n_{\mathrm{s}}=\mu_{\mathrm{r}}=\varepsilon_{\mathrm{r}}=\frac{1}{\mathrm{j} \beta_{0} d} \tanh ^{-1}\left(\frac{Z_{\mathrm{S}}}{Z_{0}}\right),
$$

which suggests that the real and imaginary parts of the complex permeability can be expressed as [15]:

$$
\begin{aligned}
& \mu_{\mathrm{r}}^{\prime}=\operatorname{Re}\left\{\frac{1}{j \beta_{0} d} \tanh ^{-1}\left(\frac{Z_{\mathrm{S}}}{Z_{0}}\right)\right\}, \\
& \mu_{\mathrm{r}}^{\prime \prime}=\operatorname{Im}\left\{\frac{1}{\mathrm{j} \beta_{0} d} \tanh ^{-1}\left(\frac{Z_{\mathrm{S}}}{Z_{0}}\right)\right\} .
\end{aligned}
$$

These equations relate the permeability (and permittivity) of the homogeneous magneto-dielectric material to the surface impedance of the composite metamaterial structure.

Finally, utilizing (3.3), it is possible to derive independent equations for $R_{\mathrm{S}}$ and $X_{\mathrm{S}}$ in terms of $\mu_{\mathrm{r}}^{\prime}$ and $\mu_{\mathrm{r}}^{\prime \prime}$ :

$$
\begin{aligned}
& R_{\mathrm{S}}=\frac{Z_{0}}{2} \frac{\sinh \left(2 \beta_{0} d \mu_{\mathrm{r}}^{\prime \prime}\right)}{\cosh ^{2}\left(\beta_{0} d \mu_{\mathrm{r}}^{\prime \prime}\right)-\sin ^{2}\left(\beta_{0} d \mu_{\mathrm{r}}^{\prime}\right)}, \\
& X_{\mathrm{S}}=\frac{Z_{0}}{2} \frac{\sinh \left(2 \beta_{0} d \mu_{\mathrm{r}}^{\prime}\right)}{\cosh ^{2}\left(\beta_{0} d \mu_{\mathrm{r}}^{\prime \prime}\right)-\sin ^{2}\left(\beta_{0} d \mu_{\mathrm{r}}^{\prime}\right)} .
\end{aligned}
$$

\subsection{Resonances at interphase boundary}

Employing the FE free energy density for the tetragonal phase of $\mathrm{BaTiO}_{3}$ [16], for zero $E$-field, we have

$$
F_{\mathrm{FE}}=\frac{\alpha}{2} P_{z}^{2}+\frac{\beta}{4} P_{z}^{4}
$$

and the FM free energy density for a uniaxial crystal in an external magnetic field

$$
F_{\mathrm{FM}}=-\frac{K_{1}}{M_{\mathrm{S}}^{2}}\left(M_{z}\right)^{2}-\mu_{0} M \times H_{\Sigma}(t) .
$$

The position of the spectral peak for the case of zero ME coupling follows from [17] $\left.\frac{1}{\gamma} \omega\right|_{\text {res }}(\delta=0)=$ $\left.\mu_{0} H^{\mathrm{LEF}}\right|_{\text {res }}=\frac{2 K_{1}}{M_{\mathrm{s}}^{2}} M_{z}+\mu_{0} H_{\mathrm{res}}$ according to which $\mu_{0} H_{\text {res }}(\delta=0) \approx 0.087 \mathrm{~T}$, and for $E \neq 0$ :

$$
\mu_{0} H_{\mathrm{res}}=\left.\frac{1}{\gamma} \omega\right|_{\mathrm{res}}(\delta \neq 0)-\frac{2 K_{1}}{M_{\mathrm{s}}^{2}} M_{z}+\delta P_{z} .
$$

This relation reveals that the resonance condition depends on the orientation of the surface polarization. In the above $\delta$ is the ME coupling coefficient.

\subsection{The case of magneto-dielectric Jaumann-type absorber}

Each layer is defined by three parameters: the thickness $\left(d_{n}\right)$, complex permittivity $\left(\varepsilon_{n}\right)$, and permeability $\left(\mu_{n}\right)$.

A recursion formula is used to calculate the reflectivity at the air/absorber interface. In this strategy the layers are numbered 1 to $n$ starting at the first layer next to the $\mathrm{PEC}$, and the interfaces are numbered 0 to $n$ starting at the PEC/first layer interface (Fig. 1).

The recursion formula, expressed below, starts by calculating the reflectivity from interface $i=1,2,3, \ldots n$, where at interface $n$ the reflectivity coefficient from the whole absorber, is obtained [14]: 


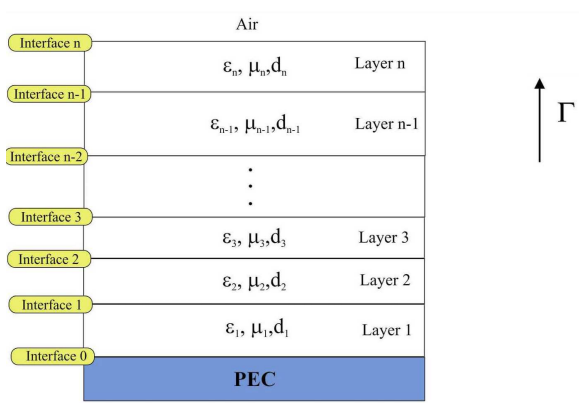

Fig. 1. Reflectivity from Jaumann absorber layers.

$$
\Gamma_{i}=\frac{\tilde{\Gamma}_{i}+\Gamma_{i-1} \mathrm{e}^{-2 \mathrm{j} k_{i-1} d_{i-1}}}{1+\tilde{\Gamma}_{i} \Gamma_{i-1} \mathrm{e}^{-2 \mathrm{j} k_{i-1} d_{i-1}}} \quad \text { for } \quad i>0,
$$

where $k_{i}$ is the component of the wave vector normal to the interface,

$$
k_{i}=\omega \sqrt{\mu_{i} \varepsilon_{i}-\sin ^{2} \theta_{i}} .
$$

$\tilde{\Gamma}_{i}$ is the reflection coefficient from interface $i$, and is dependent on the polarization such that [14]:

$$
\begin{gathered}
\tilde{\Gamma}_{i}^{\mathrm{TE}}=\frac{\mu_{i-1} k_{i}-\mu_{i} k_{i-1}}{\mu_{i-1} k_{i}+\mu_{i} k_{i-1}} \quad \text { for } \quad i>0, \\
\tilde{\Gamma}_{i}^{\mathrm{TM}}=\frac{\varepsilon_{i} k_{i-1}-\varepsilon_{i-1} k_{i}}{\varepsilon_{i} k_{i-1}+\varepsilon_{i-1} k_{i}} \quad \text { for } \quad i>0 .
\end{gathered}
$$

For the PEC/first layer interface,

$$
\tilde{\Gamma}_{i}^{\mathrm{TE} / \mathrm{TM}}=-1 \text { for } i=0 .
$$

\subsection{Some physical limitations relating to thickness}

In the paper [18] the ultimate thickness to bandwidth ratio of radar absorbers is discussed. Based simply on the assumption of linear materials and the principle of causality, the following result was obtained:

$$
\int_{0}^{\infty} \ln \left(\frac{1}{|\Gamma(\lambda)|}\right) \mathrm{d} \lambda \leq 2 \pi^{2} \sum_{i} \mu_{s, i} d_{i},
$$

where $\mu_{s, i}$ is the static relative permeability of layer $i$ and $d_{i}$ is the thickness of the corresponding layer. For nonmagnetic media, the right-hand side is simply $2 \pi^{2} d$, where $d$ is the total thickness of the absorber.

This relation states that the reflection coefficient can be small only in a limited band. This can be estimated by choosing a reflection profile which is constant $\Gamma_{0}$ in the band $\lambda_{1}<\lambda<\lambda_{2}$, indicating (for non-magnetic absorbers)

$$
\ln \left(\frac{1}{\left|\Gamma_{0}\right|}\right)\left(\lambda_{2}-\lambda_{1}\right) \leq 2 \pi^{2} d
$$

This can be expressed as a bandwidth in frequency through $\lambda_{2}-\lambda_{1}=c / f_{2}-c / f_{1}=c\left(f_{1}-f_{2}\right) / f_{1} f_{2}$.

\section{New materials in RAM}

The development of microfabrication technology in recent years has made possible, particularly in two dimensions, the controlled design of novel material composites on scales down to tens of angstroms. Motivated by such advances, entirely new classes of novel materials with superior electromagnetic properties in the microwave/ millimeter wave range have been recently proposed, including materials with voltage tunable dielectric permittivity, ultrahigh dielectric materials (e.g., carbon nanotubes), photonic band-gap materials, antiferroelectrics, magneto-dielectrics, and also some metamaterials.

To achieve a broadband performance a multi-layered absorbing structure containing a combination of various absorbing materials can be designed [15]. Each layer then would have a unique set of electromagnetic properties causing an absorption resonance at a certain frequency. However, the "hybrid" RAMs suffer from their thickness and weight. Nanoscale structures with their high surface-to-volume ratio and adjustable electromagnetic properties can be potentially used to resolve the trade-off [19].

Composites based on polymers and carbon nanofillers have gained notable attention of researches due to their high mechanical, electrical, and thermal properties at relatively low concentrations. Due to the outstanding electrical properties of carbon nanotubes (CNT), such composites are ascribed to the high aspect ratio which is generally in the range 10-1000. Such high aspect ratio enable electrical percolation of the CNT within polymers to occur at very low contents.

To a large extent, the theoretical and experimental efforts designed to provide microwave absorbers are associated with two important features of their electromagnetic properties. The first is based on enhanced magnetic losses due to, for example, resonance phenomenon in the higher frequency region ( $>300 \mathrm{MHz}$ ). It has also been argued that the second feature is related to enhanced dielectric losses. Other forms of C-based absorbers with good absorption characteristics have currently appeared, due to advances in synthesis techniques, and optimized design approaches [20-23]. Most recently, graphene joined the $\mathrm{C}$ family for microwave absorbing applications [24].

Also materials with multiferroic (magnetic, electric, and/or elastic) orders, called multiferroics (MF), have attracted increased attention again [25-28], mainly due to the discovery that the notoriously small multiferroic coupling in bulk matter may well be increased by a controlled engineering of low dimensional compounds, opening thus the way for the design of qualitatively new device concepts [29-31]. Interesting new possibilities for devicing microwave absorbing materials are offered by so called seignettomagnets (SM), a relatively new family of materials combining ferro-, antiferro-, or ferrielectric properties with ferro-, antiferro-, or ferrimagnetic properties in a certain temperature range [32].

\section{Conclusions}

In the discussion presented above we tried to revisit some problems, which in our opinion, have potential to influence, in a greater or lesser degree, the radar absorbing technology. 
The overview presented above deals with chosen problems of interest in RAM techniques, and by no means does not claim to be a complete coverage of the topic.

We focused on several points, which can be of use as design elements or even some building blocks of analytical treatment, together with trying to get some insight into physical mechanisms of actual or potential importance in an optimization of radar absorbing structures. The main emphasis was paid on complex superlattices, as exemplified by planar magneto-dielectric composites which, in our opinion, have potential for providing new design structures even without PEC, being essentially capable to enhance microwave absorption. Some analogous property presents carbon-based nanomultilayers including those using graphene in various structural configurations. We also draw particular attention to resonance absorption, of different physical origins, especially in many phase composite configurations such as antiferromagnetic (being distinctive for resonances in $\mathrm{GHz}$ range) or ferroic superlattices.

The separate role of resonances seems to be not fully realized by most of researchers, and still needs deeper analysis, also at first principles level.

\section{References}

[1] Yu.K. Kovneristyi, I.Yu. Lazareva, A.A. Ravaev, Microwave-Absorbing Materials, Nauka, Moscow 1982 (in Russian).

[2] C.R. Paul, Introduction to Electromagnetic Compatibility, 2nd ed., Wiley-Interscience, Hoboken 2006.

[3] S. Kunihiro, USA Patent 3623099, 1970.

[4] Jpn. Patent 54 (1979).

[5] E.F. Knott, C.D. Lunden, IEEE Trans. Antennas Propag. 43, 1339 (1995).

[6] J. Kapelewski, Acta Phys. Pol. A 120, 647 (2011).

[7] K.J. Vinoy, R.M. Jha, Radar Absorbing Materials: From Theory to Design and Characterization, Kluwer, Boston 1996.

[8] Z. Bayraktar, M.D. Gregory, X. Wang, D.H. Werner, IEEE Trans. Antennas Propag. 60, 1910 (2012).

[9] J. Smit, H.P.J. Wijn, Ferrites: Physical Properties of Ferromagnetic Oxides in Relation to Their Technical Applications, Philips Technical Library, Eindhoven 1959.

[10] B. Lax, K.J. Button, Microwave Ferrites and Ferrimagnetics, McGraw-Hill, New York 1962.

[11] C.P. Neo, V.K. Varadan, IEEE Trans. Electromagn. Compat. 46, 102 (2004).
[12] S. Chikazumi, Physics of Ferromagnetism, Oxford University Press, Oxford 1997.

[13] T. Nagamiya, K. Yosida, R. Kubo, Adv. Phys. 4, 1 (1955).

[14] P. Saville, Review of Radar Absorbing Materials, Defence R\&D Canada, Atlantic 2005.

[15] E. Knott, J. Shaeffer, M. Tulley, Radar Cross Section, 2nd ed., SciTech Publ., New York 2004.

[16] A. Sukhov, C.-L. Jia, P.P. Horley, J. Berakdar, J. Phys., Condens. Matter 22, 352201 (2010).

[17] C. Kittel, Phys. Rev. 73, 155 (1948).

[18] K.N. Rozanov, IEEE Trans. Antennas Propagat. 48, 1230 (2000).

[19] J. Cao, W. Fu, H. Yang, Q. Yu, Y. Zhang, S. Liu, P. Sun, X. Zhou, Y. Leng, S. Wang, B. Liu, G. Zou, J. Phys. Chem. B 113, 4642 (2009).

[20] A.-Y. Ge, B.-S. Xu, X.-M. Wang, T.-B. Li, P. Han, X.-G. Liu, Acta Phys. Chim. Sin. 22, 203 (2006).

[21] D.S. Bychanok, S.I. Moseenkov, V.L. Kuznetsov, P.P. Kuzhir, S.A. Maksimenko, K.G. Batrakov, O.V. Ruhavets, A.V. Gusinski, O. Shenderova, $\mathrm{Ph}$. Lambin, J. Nanoelectron. Optoelectron. 4, 257 (2009).

[22] Q.-W. Chen, Ch. Yao, C. Fangyu, rth IEEE Conf. on Nanotechnology, Hong Kong 2007, p. 1326.

[23] Y. Bai, J. Zhou, Z. Gui, L. Li, L. Qiao, J. Appl. Phys. 101, 083907 (2007).

[24] J.-J. Fang, S.-F. Li, W.-K. Zha, H.-Y. Cong, J.-F. Chen, Z.-Z. Chen, J. Inorg. Mater. 26, 467 (2011).

[25] W. Eerenstein, N.D. Mathur, J.F. Scott, Nature 442 , 759 (2006).

[26] M. Fiebig, J. Phys. D 38, R123 (2005).

[27] N. Spaldin, M. Fiebig, Science 309, 391 (2005).

[28] R. Ramesh, N.A. Spaldin, Nature Mater. 6, 21 (2007).

[29] M. Bibes, A. Barthelemy, Nature Mater. 7, 425 (2008).

[30] C.A.F. Vaz, J. Hoffman, C.H. Ahn, R. Ramesh, Adv. Mater. 22, 2900 (2010).

[31] F. Zavaliche, T. Zhao, H. Zheng, F. Straub, M.P. Cruz, P.-L. Yang, D. Hao, R. Ramesh, Nano Lett. 7, 1586 (2007).

[32] D.I. Mirovitskii, V.M. Petrov, Radiotekhnika 3, 60 (1989). 